\title{
Recurrent myocardial infarction secondary to Prinzmetal's variant angina
}

\author{
Dale $\underline{\text { Murdoch }}^{1,2}$, MBBS, FRACP, Priyanka $\underline{\text { Dhillon }}^{1}$, MBBS, Selvanayagam Niranjan ${ }^{1,3}$, FRACP
}

\begin{abstract}
Prinzmetal's variant angina describes chest pain secondary to reversible coronary artery vasospasm in the context of both diseased and non-diseased coronary arteries. Symptoms typically occur when the patient is at rest and are associated with transient ST-segment elevation. Acute episodes respond to glyceryl trinitrate, but myocardial infarction and other potentially fatal complications can occur, and long-term management can be challenging. Although it is not well understood, the underlying mechanism appears to involve a combination of endothelial damage and vasoactive mediators. In this case, a 35-year-old woman with myocardial infarction secondary to coronary artery vasospasm experienced recurrent chest pain. Coronary angiography revealed severe focal stenosis in the mid left anterior descending artery, which completely resolved after administration of intracoronary glyceryl trinitrate. The patient was discharged on nitrates and calcium channel blockers. The patient re-presented with another myocardial infarction, requiring up-titration of medical therapy.
\end{abstract}

Keywords: coronary artery spasm, myocardial infarction, Prinzmetal's variant angina, vasospastic angina

\section{INTRODUCTION}

Prinzmetal's variant angina (PVA) is an infrequent syndrome in which myocardial ischaemia is heralded by chest pain when a person is at rest, typically during early morning, and transient ST-segment elevation occurs due to reversible coronary artery vasospasm. ${ }^{(1-4)}$ This phenomenon, which is relieved by glyceryl trinitrate $(\mathrm{GTN})$, can occur in diseased and non-diseased coronary arteries. ${ }^{(1,2)}$ In 1959, Dr Myron Prinzmetal hypothesised that variant angina was the result of vasospasm at the site of atherosclerotic lesions. ${ }^{(1)}$ Since then, coronary angiography has demonstrated that myocardial ischaemia due to coronary vasospasm can occur in the context of normal coronary arteries without macroscopic evidence of atherosclerosis or occlusion. . $^{(3,5,6)}$ However, accurate clinical, biochemical and electrocardiograph PVA diagnoses during patient presentation remain elusive, as the syndrome can mimic a number of pathologies, including ischaemic heart disease secondary to plaque rupture (type I acute myocardial infarction), takotsubo cardiomyopathy, pericarditis and pulmonary embolism. ${ }^{(3,5)}$ The problem of delayed diagnosis is often compounded by the transient nature of PVA. Although rare, PVA is associated with myocardial infarction, ventricular arrhythmias and death. ${ }^{(6,7)}$ The case presented herein demonstrates the difficulty in diagnosis and clinical management of PVA.

\section{CASE REPORT}

A 35-year-old woman woke from sleep with central 'squeezing' chest pain that radiated to her back and was associated with leftarm paraesthesia. The pain was not pleuritic in character. The patient was dyspnoeic and experienced palpitations and nausea, and vomited once. She reported two episodes of chest pain that were ten minutes apart and of approximately ten minutes' duration. The patient had been mildly unwell with an upper respiratory tract infection for seven days prior to this. She did not, however, have any infectious symptoms at presentation. Upon admission, she experienced another episode of chest pain in the emergency department, which resolved with GTN and intravenous morphine.

The patient's medical history revealed previous radiofrequency catheter ablation for supraventricular tachycardia in 2005. She did not have hypertension, dyslipidaemia or diabetes mellitus. She was an ex-smoker who had quit eight years prior to presentation. The family history was significant, as her father had died of an acute myocardial infarction at the age of 40 . His autopsy report demonstrated advanced coronary artery atherosclerosis with acute myocardial infarction.

The patient was not on any regular medication. On examination, her vitals were stable (heart rate $60 \mathrm{bpm}$; blood pressure $115 / 70$; saturating at $100 \%$ on $2 \mathrm{~L}$ of oxygen; respiratory rate 16 breaths per minute; temperature $37.4^{\circ} \mathrm{C}$ ). Heart sounds were dual with no murmurs. Jugular venous pressure was not elevated and there was no peripheral oedema. Chest sounds were normal on auscultation and an abdominal examination was unremarkable.

Initial electrocardiography (ECG) revealed no ischaemic changes in sinus rhythm (Fig. 1a) and cardiac troponin I (cTnl) level that was not elevated at $0.02 \mu \mathrm{g} / \mathrm{L}$ (reference range $<0.04 \mu \mathrm{g} / \mathrm{L}$ ). Electrolytes, full blood count and lipids were normal. The patient was admitted to the coronary care unit where her ongoing anginal chest pain required escalation of therapy. Serial ECGs demonstrated anterior ST-segment elevation during chest pain and subsequently deep, symmetrical T-wave inversion in leads V1-3 (Fig. 1b \& c). A repeat test after eight hours showed that the patient's cTnl level was elevated at $0.3 \mathrm{ng} / \mathrm{L}$ (reference range $<0.04 \mathrm{ng} / \mathrm{L}$ ).

Initial coronary angiography in the left anterior oblique cranial projection showed a tapering $90 \%$ mid left anterior descending (LAD) stenosis just after the first diagonal artery. Intracoronary GTN was administered and the focal LAD stenosis disappeared completely (Fig. 2). A left ventriculogram showed a focal area of 

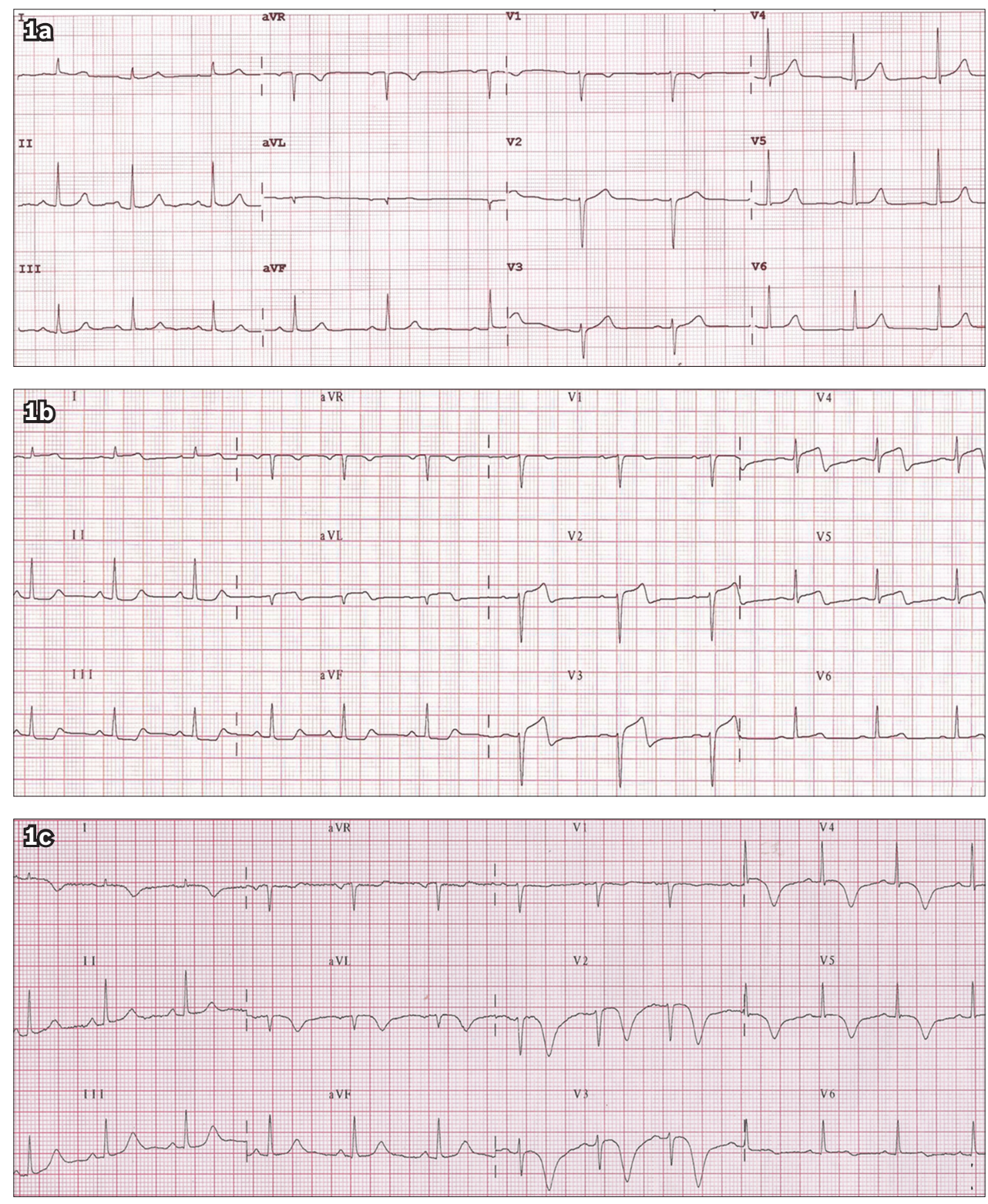

Fig. 1 Serial ECGs show (a) an initially normal ECG; (b) anterior ST-segment elevation recorded at the time of pain; and (c) subsequent deep, symmetrical T-wave inversion indicating myocardial ischaemia/infarction.
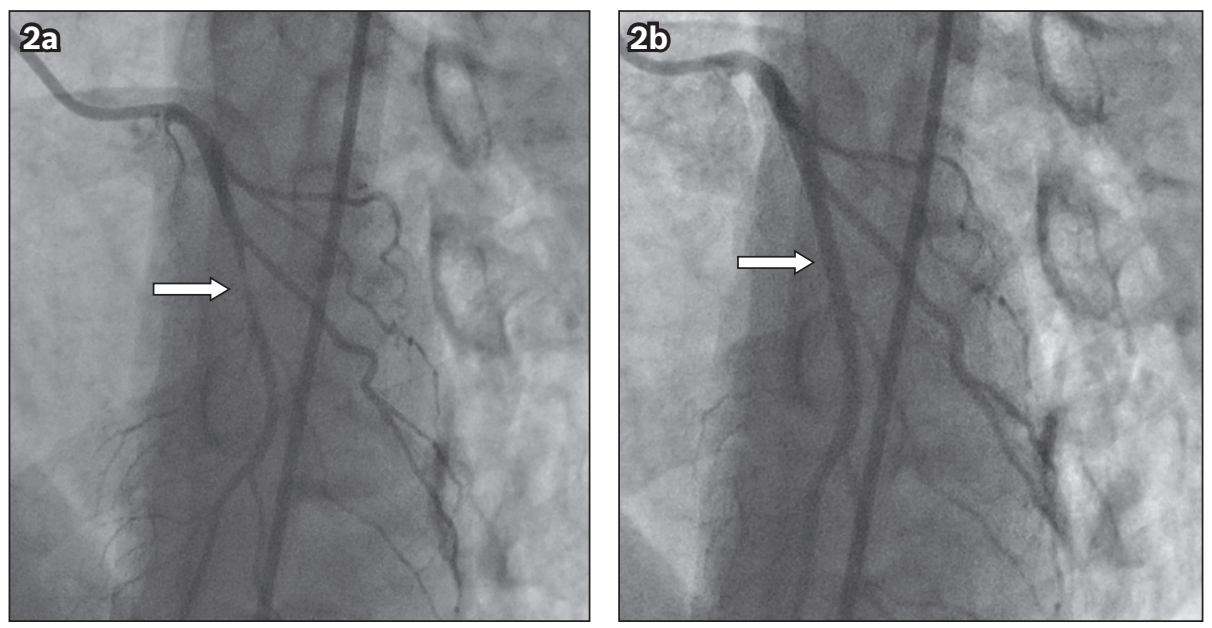

Fig. 2 Diagnostic coronary angiograms show (a) severe stenosis in the mid left anterior descending artery (arrow) on the initial injection; and (b) complete resolution of stenosis (arrow) after administration of $200 \mathrm{mcg}$ of intracoronary glyceryl trinitrate. 
anterolateral hypokinesia. A diagnosis of coronary artery spasm (PVA) with secondary acute myocardial infarction was made.

Cardiac magnetic resonance imaging demonstrated that leftventricular and right-ventricular size and function was normal with no valvular abnormality. There was no left-ventricular wall oedema to suggest myocarditis and no evidence of significant myocardial scarring. The patient was treated with diltiazem sustained-release (SR) $240 \mathrm{mg}$ daily, isosorbide mononitrate SR $60 \mathrm{mg}$ daily and GTN spray as required. She was discharged from hospital with follow-up arrangements in place.

Five months after her initial presentation, the patient presented with four distinct episodes of central crushing pain in the chest that radiated to the left arm. The pain was associated with vomiting and diaphoresis, and was not relieved by GTN. Once again, ECGs demonstrated $\mathrm{T}$-wave inversion in $\mathrm{V} 1-3$. A repeat test showed that cTnl level was elevated at $0.4 \mathrm{ng} / \mathrm{L}$. The patient continued to experience chest pain while on the ward, and telemetry with ST-segment monitoring showed transient ST-segment elevation (Fig. 3). Her dose of isosorbide mononitrate SR was increased to $90 \mathrm{mg}$ daily and nicorandil of $10 \mathrm{mg}$ daily was added. Computed tomography coronary angiography that was performed after the administration of GTN demonstrated no coronary artery occlusion or calcification. The patient responded well to the adjustments in her medication and her symptoms were under control at one-year follow-up.

\section{DISCUSSION}

PVA is recognised as chest pain not precipitated by physical exertion and is associated with ST-segment elevation. ${ }^{(1-4)}$ It is an important functional cardiac disorder associated with syncope, high-degree atrioventricular block, life-threatening arrhythmias, acute myocardial infarction and cardiac arrest. ${ }^{(2,7,8)}$ Typically, chest pain secondary to PVA occurs in the early hours of the morning and responds well to GTN. ${ }^{(2,7)}$ The symptoms are the result of vasospasm in normal or atherosclerotic cardiac vessels. Approximately $30 \%$ of patients who undergo coronary angiography in suspected acute coronary syndrome have no culprit lesion or have disease-free coronary arteries, ${ }^{(3)}$ and while some of these patients will have PVA, the exact proportion is unknown. A diagnosis can be confidently made by recording ST-segment elevation at the time of chest pain, but capturing these events can be difficult. Therefore, the use of telemetry or Holter monitoring with ST-segment monitoring is recommended. Provocation testing using intracoronary acetylcholine is safe and effective in demonstrating coronary artery vasospasm. ${ }^{(3)}$

Although the exact aetiology of coronary vasospasm remains unclear, several mechanisms have been hypothesised. ${ }^{(5)}$ A combination of arterial wall dysfunction and vasoactive substances are thought to be involved. Primarily endothelial damage exposes arterial media to vasoconstrictors such as catecholamines, thromboxane A2, serotonin, endothelin and histamine. ${ }^{(2,9)}$ Reports also suggest that an imbalance in local vasodilators such as prostacyclin and nitric oxide increases the hyperreactivity of cardiac arteries. ${ }^{(2)}$ In addition, endothelial nitric oxide synthase T-786C mutation has been associated with increased coronary

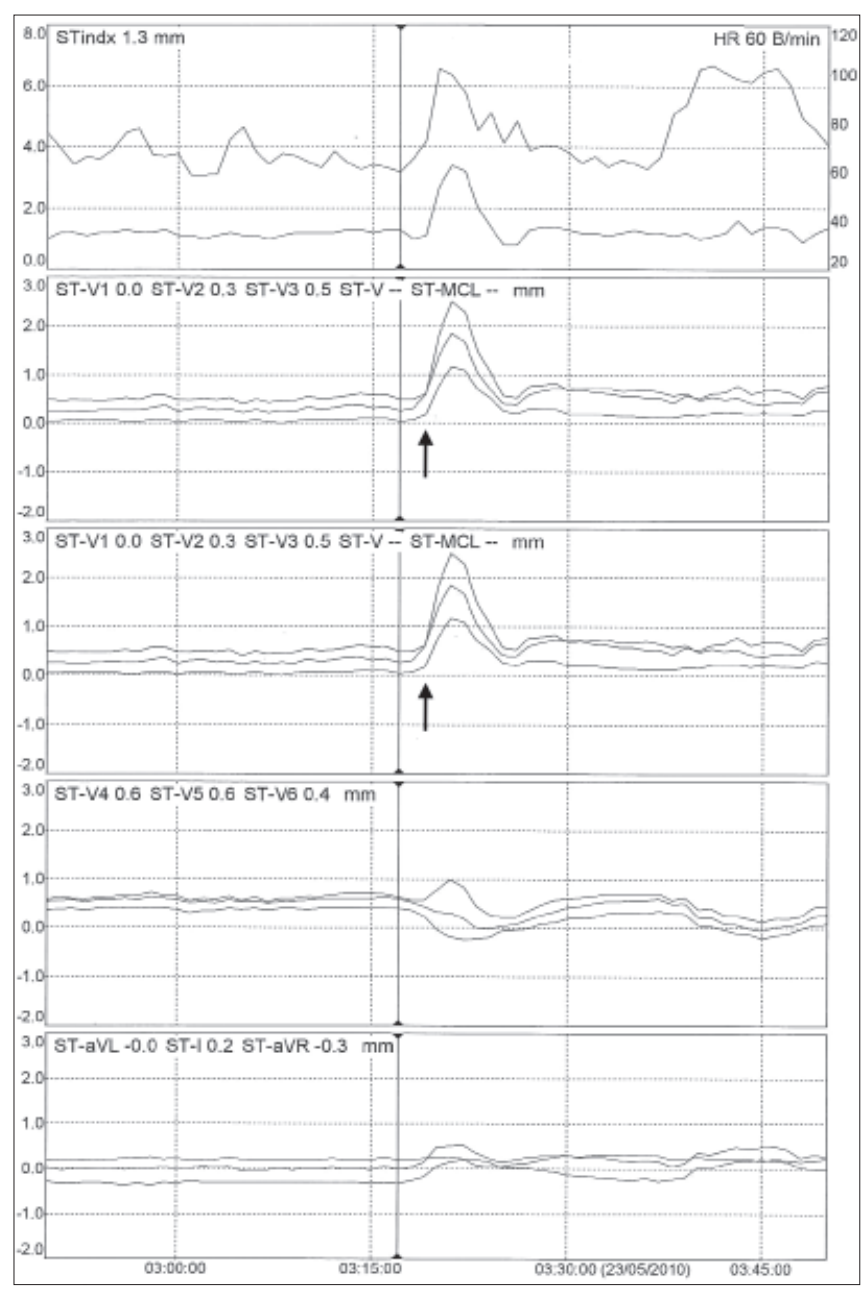

Fig. 3 Telemetry with ST-segment monitoring shows significant ST-segment elevation corresponding with episodes of pain (arrows) in leads V1, V2 and V3.

artery basal tone due to a reduction in nitric oxide production. ${ }^{(10)}$ This, in turn, increases vessel susceptibility to vasoconstrictors such as acetylcholine and predisposition to coronary artery vasospasm. ${ }^{(10,11)}$

There appears to be a higher incidence of PVA in Asian countries as opposed to Caucasian populations. ${ }^{(2-4,12)}$ Miwa et al demonstrated a positive result in $93 \%$ of Japanese patients who underwent provocation testing, ${ }^{(13)}$ while a prevalence of $74 \%$ was found in a Taiwanese population. ${ }^{(14)}$ The CASPAR (coronary artery spasm in patients with acute coronary syndrome) study conducted in a Caucasian study group, in comparison, had a prevalence of coronary spasm of $49 \% .{ }^{(3)}$ The disparity may be due to more diffuse involvement of coronary arteries, hyperreactivity and increased basal tone in the Japanese population. ${ }^{(3)}$

Current treatment involves the use of calcium channel blockers, nitrates, nicorandil and fasudil. Dose titration and addition of other medications are often required. Long-acting calcium channel blockers have been shown to decrease the number of anginal attacks and are considered the mainstay of treatment. ${ }^{(4,15)}$ For this reason, they are used routinely in the treatment of hypertension in Japan. In addition to medical management, smoking cessation correlates with a reduction in symptoms. 
Table I. The Japanese Coronary Spasm Association clinical risk score for patients with vasospastic angina. ${ }^{(12)}$

\begin{tabular}{lc}
\hline Predictive factor & Score $^{*}$ \\
\hline History of OHCA & 4 \\
Smoking & 2 \\
Angina at rest alone & 2 \\
Significant organic stenosis & 2 \\
Multivessel spasm & 2 \\
ST elevation & 1 \\
Beta-blocker use & 1 \\
\hline
\end{tabular}

*Low risk $0-2$, intermediate risk $3-5$, high risk $\geq 6$, out of a possible range of 0-14. OHCA: out-of-hospital cardiac arrest

The overall prevalence of myocardial infarction with normal coronary angiography is approximately $3 \%$ and tends to be more common in younger patients. ${ }^{(5)}$ Several studies that attempted to identify prognostic features of Prinzmetal's angina consistently indicated that smoking, coronary artery disease and multivessel disease were poor prognostic factors. Takagi et al developed the Japanese Coronary Spasm Association clinical risk score for patients with vasospastic angina. Patients' risk of having major adverse cardiac events (MACE) can be evaluated with this novel scoring system, in which low-, intermediate- and high-risk categories have MACE rates of $2.5 \%, 7 \%$ and $13 \%$, respectively. The authors identified seven significant predictors of a major adverse cardiac event, including smoking, angina at rest, outof-hospital cardiac arrest, significant organic coronary stenosis, multivessel spasm, ST elevation and beta-blocker use (Table I). ${ }^{(12)}$ Using these predictors, the group categorised risk-stratified patients into three groups, with a prediction rate of approximately $90 \% .{ }^{(12)}$ Da Costa et al identified left ventricular ejection fraction, age, smoking and diabetes as predictors for poor prognosis in patients with myocardial infarction and normal coronary arteries, while multivariate analysis only identified left ventricular function and diabetes mellitus. ${ }^{(5)}$ Similarly, the CASPAR study only identified left ventricular function as an independent predictor of poor prognosis in patients with coronary vasopsasm. ${ }^{(3)}$

In conclusion, PVA remains a difficult diagnosis and management dilemma, and strong clinical suspicion and careful history-taking are paramount. Although the lack of diseased coronary vessels mitigates the risk of myocardial infarction to some extent, myocardial infarction is nevertheless a very real possibility, as was demonstrated in this case, and thus should not be underestimated. Patient risk factors and prognostic factors do not fundamentally allow for differentiation between acute coronary syndrome due to atherosclerotic disease and that which occurs in normal arteries. Although several attempts have been made, more research is required into management guidelines for PVA.

\section{REFERENCES}

1. Prinzmetal M, Kennamer R, Merliss R, Wada T, Bor N. Angina pectoris. I. A variant form of angina pectoris; preliminary report. Am J Med 1959; 27:375-88.

2. Mayer S, Hillis LD. Prinzmetal's variant angina. Clin Cardiol 1998; 21:243-6.

3. Ong P, Athanasiadis A, Hill S, et al. Coronary artery spasm as a frequent cause of acute coronary syndrome: The CASPAR (Coronary Artery Spasm in Patients With Acute Coronary Syndrome) Study. J Am Coll Cardiol 2008; 52:523-7.

4. Stern S, Bayes de Luna A. Coronary artery spasm: a 2009 update. Circulation 2009; 119:2531-4.

5. Da Costa A, Isaaz K, Faure E, et al. Clinical characteristics, aetiological factors and long-term prognosis of myocardial infarction with an absolutely normal coronary angiogram; a 3-year follow-up study of 91 patients. Eur Heart J 2001; 22:1459-65.

6. Ong P, Athanasiadis A, Borgulya G, Voehringer M, Sechtem U. 3-year follow-up of patients with coronary artery spasm as cause of acute coronary syndrome: the CASPAR (coronary artery spasm in patients with acute coronary syndrome) study follow-up. J Am Coll Cardiol 2011; 57:147-52.

7. Chin A, Casey M. Variant angina complicated by polymorphic ventricular tachycardia. Int J Cardiol 2010; 145:e47-9.

8. Bohm A, Kiss R, Dorian P, Pinter A. Complications of variant angina: a case report. Can J Cardiol 2012; 28:245.e5-7.

9. Willerson JT, Hillis LD, Winniford M, Buja LM. Speculation regarding mechanisms responsible for acute ischemic heart disease syndromes. J Am Coll Cardiol 1986; 8:245-50.

10. Glueck C, Munjal J, Khan A, Umar M, Wang P. Endothelial nitric oxide synthase T-786C mutation, a reversible etiology of Prinzmetal's angina pectoris. Am J Cardiol 2010; 105:792-6.

11. Yoshimura $M$, Yasue $H$, Nakayama $M$, et al. Genetic risk factors for coronary artery spasm: significance of endothelial nitric oxide synthase gene T-786-->C and missense Glu298Asp variants. J Investig Med 2000; 48:367-74.

12. Takagi Y, Takahashi J, Yasuda S, et al; Japanese Coronary Spasm Association. Prognostic stratification of patients with vasospastic angina: a comprehensive clinical risk score developed by the Japanese Coronary Spasm Association. J Am Coll Cardiol 2013; 62:1144-53.

13. Miwa K, Fujita M, Ejiri M, Sasayama S. The sensitivity of intracoronary injection of acetylcholine in inducing coronary spasm differs in patients with stable and unstable angina. Int J Cardiol 1992; 36:329-39.

14. Wang CH, Kuo LT, Hung MJ, Cherng WJ. Coronary vasospasm as a possible cause of elevated cardiac troponin I in patients with acute coronary syndrome and insignificant coronary artery disease. Am Heart J 2002; 144:275-81.

15. Sueda S, Kohno H, Fukuda H, Uraoka T. Did the widespread use of longacting calcium antagonists decrease the occurrence of variant angina? Chest 2003; 124:2074-8. 